\title{
Mineral Derangement and Bone Diseases in Uremic Patients on hemodialysis in Ibn-Sina Hemodialysis Center / Diyala Shakir Hameed Mohamed (FICMS) ${ }^{1}$, Ahmed Methab Athab $(\mathrm{PhD})^{2}$, Nabeel Khalid mohammed Ali (FICMS) ${ }^{3}$ and Ismail Ibrahim Latif (MbC B, PhD,HMD $)^{4}$
}

\section{Abstract}

Background: Chronic kidney disease is an international public health problem affecting 5$10 \%$ of the world population. As kidney function declines, there is a progressive deterioration in mineral homeostasis, with a disruption of normal serum and tissue concentrations of phosphorus and calcium, and changes in circulating levels of hormones. Objective: To determine the severity of bone and mineral disease in chronic renal failure and how efficiently controlled by drugs and hemodialysis procedure in Ibn sina dialysis center.

Patients and Methods: A case control study was carried out among 75 patients with chronic kidney disease (stage5) on regular hemodialysis in Ibn Sina dialysis center. Blood was taken before dialysis session and measurements of serum calcium, phosphorus, serum alkaline phosphatase, .protein and albumin levels, Serum parathyroid hormone and VIT D3 were determined. and another group of 25 normal peoples from surrounding area.

Results: The mean parathyroid hormone level was $145.62 \mathrm{pg} / \mathrm{ml}$, with $57.33 \%$ having normal acceptable rang of parathormone level, $41 \%$ of patients having hyperparathyroidism and $1.33 \%$ having hypoparathyroidism. There was no significant correlation between serum PTH and dexa scan. Hypocalcemia was found in $52 \%$ of patients, $33 \%$ with hypercalcemia patients . There was a significant correlation found between Hypocalcemia and dexa scan results. Majority of patients had high serum phosphate levels (54.6\%).and normal phosphate level in $40 \%$ while the hypophosphatemia result $5.33 \%$ and mean phosphate level is $2.24 \mathrm{mmol} / \mathrm{l}$.Total serum protein mean is 61.51 a $37.33 \%$ of them had hypoproteinemia and $62.66 \%$ of them had normal serum protein. Also our results show 97, 33\% of uremic patients have low vit D While $2.33 \%$ have normal level of serum vit $\mathrm{D}$. There was a significant correlation between dexa scan and vit $\mathrm{D}$ level, calcium, alkaline phosphatase, female gender, smoking and body mass index.

Conclusion: The current study demonstrated that we can predict the chronic kidney diseasesMetabolic bone disorder from mineral scores before potentates the diagnosis by dexa scan. Keywords: Metabolic bone disorder, dexa scan, chronic kidney disease Corresponding Author: ismail_6725@yahoo.com

Received: $30^{\text {th }}$ April 2019

Accepted: $27^{\text {th }}$ May 2019

\footnotetext{
${ }^{1,3}$ Al- Batool Teaching Hospital-Diyala-Iraq.

${ }^{2,4}$ College of Medicine - University of Diyala -Diyala-Iraq.
} 


\section{Introduction}

Chronic kidney disease (CKD) is a worldwide public health problem, with increasing prevalence[1]. It is associated with a number of abnormalities in mineral metabolism viz. hypocalcemia, hyperphosphatemia and abnormalities in vitamin $\mathrm{D}$ metabolism that result in functional calcitriol deficiency[2,3,4]. The major consequences of disordered mineral metabolism in CKD are secondary hyperparathyroidism (SHPT), metabolic bone disease including renal osteodystrophy and vascular calcification[4,5,6]. These include parathyroid hormone (PTH), 25-hydroxyvitamin D (25(OH)D), 1,25-dihydroxyvitamin D $(1,25(\mathrm{OH}) 2 \mathrm{D})$, and other vitamin D metabolites, fibroblast growth factor-23 (FGF-23), and growth hormone. Beginning in CKD stage 3, the ability of the kidneys to appropriately excrete a phosphate load is diminished, leading to hyperphosphatemia, elevated PTH, and decreased 1,25(OH)2D with associated elevations in the levels of FGF-23. The conversion of $25(\mathrm{OH}) \mathrm{D}$ to $1,25(\mathrm{OH}) 2 \mathrm{D}$ is impaired, reducing intestinal calcium absorption and increasing PTH $[4,5,6]$.The treatment with vitamin $\mathrm{D}$, reduction of PTH levels and clearance of phosphate are all beneficial to the bone after hemodialysis (HD). However, bone loss still remains an important problem due to post HD factors such as filter size and dialysate fluid type used, persistent HPT, and other metabolic and acid-base disturbances [7].

The therapeutic strategies for alleviation of post HD bone disease include, low dose of calcium, vitamin D, calcimimetic agents, bisphosphonates and, to a lesser extent, calcitonin, good nutrition and avoidance of analgesic and steroid[8].

Despite the knowledge of existence of mineral disorders after hemodialysis, there are no studies describing the pattern of the disorder among hemodialysis patients in Diyala /Iraq. Aim of this study to determine the levels of bone mineral metabolism parameters among HD patients attending renal clinics at Baquba Teaching Hospital, IBN -Senna center.

\section{Specific objectives}

1-To determine the serum levels of calcium, phosphate and intact parathyroid hormone among HD patients in IBS center.

2-To determine the relationship between bone biochemical parameters and dexa scan of bones at hip and spine areas.

\section{Patients and Methods}

Study Design: It is a case control study. Study Area: Ibn- Sina hemodialysis Center (ISC) at the Baquba teaching Hospital, Diyala/Iraq. Study Population: HD patients of 20 years and above who have signed an informed consent. Study Duration: Enrollment was done from September 2016 to April 2017. Sample Size: 75 of HD patients on regular hemodialysis in Ibn Sina dialysis center 51 of them were males and 24 females aged between 20 and 78 years were studied and another 25 randomly chosen controlled normal peoples in the same territory was taken to compare results regarding dexa scan. And the size determined accordingly[9]. Patients were those with end stage renal disease (ESRD) on regular HD 
for 4 hours, three times a week, irrespective to etiology. All dexa scan were done within 2 hours before or after a dialysis session. Inclusion criteria: HD patients who are at least 3 months on HD programme and are on follow up at ISC hemodialysis in BTH. Patients 20 years old aged patients and above who have signed an informed consent. Exclusion criteria: Patients who have a bone diseases for other reasons that may affect the results.

A printed questionnaire was used to collect data from recruited patients. Data consisted of Socio-demographic data including age, gender and dwell area. Information on smoking, alcohol intake, drugs. Medical history for possible etiology of kidney disease, time on dialysis program, since HD started and current medications were recorded

\section{Laboratory Methods}

About $5 \mathrm{ml}$ of blood was drawn before the session of hemodialysis in sterile plain tube for creatinine, urea, calcium, phosphate, total serum protein, vit D3, Alkaline phosphatase, albumin and intact PTH. The measurements of serum creatinine, albumin, calcium and phosphate were determined using automated clinical chemistry analyzer (cobas 400 plus) in IBN-Sina center laboratory, while serum PTH assays were performed in Baquba teaching hospital main laboratory using Enzyme Linked Immuno Sorbent Assay (ELISA) (Cobas e 411). Selective measurement of the (mainly) intact parathyroid hormone permits direct ascertainment of the secretory activity of the parathyroid gland (10-11). The results were analyzed after daily calibration using standard calibration methods and materials and tests assayed against controls. The glomerular filtration rate (GFR) was estimated using the MDRD formula (12) $\mathrm{eGFR}=32788$ ラ Serum Creatinine-1.154 x Age-0.203 x (1.212 if Black) $x$ (0.742 if Female) the formula was calculated using a free internet-based clinical equation calculator [13].

Total serum calcium was corrected for serum albumin using the equation: Corrected Calcium $=\mathrm{Ca}$ (40-measured serum albumin) $\mathrm{x}$ $0.02+$ measured serum calcium. (A typical correction is that for every $1 \mathrm{~g} / \mathrm{l}$ that the albumin concentration is below $40 \mathrm{~g} / \mathrm{l}$, the calcium concentration is $0.02 \mathrm{mmol} / 1$ below what it would be if the albumin concentration was normal).All patients sent to Dexa scan unit in Baquba Teaching Hospital were weight,hight, gender obtained and dexa scan is done for lumber spine and hip area .It is done by dexa DMS group -stratos court model (France made) and software programme for Turkish ethnicity because nearest area to Iraqi ethnicity and share similar features and habits. Each one takes a report by the technician on it.

\section{Statistical analysis}

Data cleaning was performed at the end of data entry and analysis performed using SPSS version 20.0 software. The characteristics of the participants were summarized into means/medians and proportions for continuous and categorical variables respectively. eGFR, creatinine, 
serum calcium, phosphate, vit D, total serum protein, serum albumin, alkaline phosphatase and PTH were analyzed and presented as means and medians where appropriate. DEXA scan for all patient and controlled were also done and T._score for hip and spine area measured were classified as normal, Osteopenia and osteoporosis. Furthermore, calcium, phosphate and PTH were categorized according to patients with low, normal and high levels and presented as proportions. The relationship between bone biochemical parameters and dexa scan results was measured using Pearson correlation coefficient ( $\mathrm{r}$ ) and spearman s correlation coefficient, and linear regression coefficient $(\beta)$. The levels of PTH, calcium and phosphate were correlated with duration of dialysis, age of the patients was compared between groups using a nova test. All statistical tests were performed at $5 \%$ level of significance $(95 \%$ confidence interval).

\section{Results}

Most of our patients are male (58\%) with wide distribution of age at presentation but most of them between 50-70 (58\%) and smokers constitute $(33 \%)$ most of them are overweight $(54.7 \%)$ possibly fluid overload. Most of them are medium class regarding socioeconomic state (64\%) and living in urban area $(54.7 \%)$ and small numbers are alcoholic (8\%) Table (1).

Table (1): Socio -demographic data of study population.

\begin{tabular}{||c||c||c||c|}
\hline \multicolumn{1}{|c||}{ Gender } & & BMI & \\
\hline male & $51(68 \%)$ & normal & $23(30.7 \%)$ \\
\hline \hline female & $24(32 \%)$ & overweight & $40(54.7 \%)$ \\
\hline \hline Age & & obese & $11(14.7 \%)$ \\
\hline $20-20$ & $5(6.7 \%)$ & residence & \\
\hline $30-39$ & $11(14.7 \%)$ & rural & $34(45.3 \%)$ \\
\hline $40-49$ & $12(16 \%)$ & urban & $41(54.7 \%)$ \\
\hline $50-59$ & $27(36 \%)$ & Socioeconomic state & \\
\hline $60-69$ & $17(22.7 \%)$ & low & $22(29.3 \%)$ \\
\hline \hline $70-79$ & $3(4 \%)$ & medium & $48(64 \%)$ \\
\hline \hline Smoking & $25(33.3 \%)$ & high & $5(6.7 \%)$ \\
\hline \hline Alcohol & $6(8 \%)$ & & \\
\hline \hline
\end{tabular}

More than $(50 \%)$ are on HD for more than

among them as shown in Table (2).

2 years which indicate good survival rate

Table (2): Patient clinical characteristics.

\begin{tabular}{|c|c|}
\hline Period of dialysis (month) & \\
\hline \hline $1-3$ & $1(1.3 \%)$ \\
\hline $4-6$ & $3(4 \%)$ \\
\hline \hline $7-12$ & $13(17.3 \%)$ \\
\hline \hline $13-24$ & $20(26.7 \%)$ \\
\hline \hline More than 24 & $38(50,7 \%)$ \\
\hline
\end{tabular}


Mineral Derangement and Bone Diseases in Uremic Patients on hemodialysis in Ibn-Sina Hemodialysis

Center / Diyala

Our result showed high incidence of hypertension among patients $(81 \%)$ whether primary cause or secondary to CKD , diabetes present as cause of uremia in $(33.3 \%)$ chronic
GN in $(9.3 \%)$ yet geneto urinary infection and renal stone are present in $(33.3 \%),(26.7 \%)$ respectively Table(3).

Table (3): Past and present disease and possible causes of chronic kidney disease.

\begin{tabular}{||c||c|}
\hline \multicolumn{1}{|c||}{ Disease } & Present \\
\hline Hypertension & $61(81.3 \%)$ \\
\hline \hline diabetes & $25(33.3 \%)$ \\
\hline \hline Genitourinary & $25(33.3 \%)$ \\
\hline \hline Renal stone & $20(26.7 \%)$ \\
\hline \hline Unknown cause & $12(16 \%)$ \\
\hline \hline Chronic GN & $7(9.3 \%)$ \\
\hline \hline Bone disease & $4(5.3 \%)$ \\
\hline \hline fracture & $3(4 \%)$ \\
\hline Polycystic disease & $2(2.7 \%)$ \\
\hline \multicolumn{2}{|c|}{} \\
\hline
\end{tabular}

Most of our patients are on regular treatment of calcium carbonate $(93 \%)$ calcitriol (one alfa) $(93 \%)$ and all of them receive erythropoietin supplement and iron Table(4). (100\%).significant number have history of abuse of steroidal and non-steroidal drugs $(33.3 \%)(80 \%)$ respectively as shown in

Table (4): Shown the drug administration (past and present drugs).

\begin{tabular}{||c||c||}
\hline \multicolumn{1}{|c|}{ Drug history } & Present \\
\hline \hline steroid & $25(33.3 \%)$ \\
\hline \hline NSAID & $60(80 \%)$ \\
\hline \hline Ferro folic acid & $75(100 \%)$ \\
\hline \hline Calcium carbonate & $70(93 \%)$ \\
\hline \hline Calcitriol(one alfa) & $70(93 \%)$ \\
\hline \hline Sevelamer & $5(7 \%)$ \\
\hline \hline Epirax(erythropoietin) & $75(100 \%)$ \\
\hline
\end{tabular}

Most of our patients have normal PTH $(57.33 \%)$ and increase level of PTH in (41.33\%).regarding calcium level majority have low calcium (52\%) and normal calcium level in 46.6.phosphate are high in (55\%) and normal in $(40 \%)$.alk.phosph are normal in
(66.6\%) but elevated level present in only $(30.6 \%)$.VIT D are low in almost all patients (97.3\%).most of patients have low TSP and low Albumen (62.66\%) (74.66\%) respectively Table (5). 
Mineral Derangement and Bone Diseases in Uremic Patients on hemodialysis in Ibn-Sina Hemodialysis

Table (5): Biochemical characteristics of the study population.

\begin{tabular}{|c|c|c|c|c|}
\hline & hypo & normal & hyper & mean \\
\hline PTN & $1(1.33 \%)$ & $43(57.33 \%)$ & $31(41.33 \%)$ & $145.62( \pm 255.16)$ \\
\hline Calicium & $39(52 \%)$ & $35(46.6 \%)$ & $1(1.33 \%)$ & $2.2( \pm 0.2)$ \\
\hline Phosphate & $4(5 \%)$ & $30(40 \%)$ & $41(55 \%)$ & 2.4 \\
\hline Alk. Phosphatase & $2(2.6 \%)$ & $50(66.6 \%)$ & $23(30.6 \%)$ & 123.23 \\
\hline Vit D & $73(97.3 \%)$ & $2(2.66 \%)$ & & 38.3 \\
\hline Total serum protien & $47(62.66 \%)$ & $28(37 \%)$ & & 61.51 \\
\hline Serum albumen & $56(74.66 \%)$ & $19(25.33)$ & & 30.11 \\
\hline
\end{tabular}

Dexa scan showed that nearly half $(46.66 \%)$ of osteoporosis $(26.66 \%)$ Table (6). patients have osteopenia and only quarter have

Table (6): Dexa scan results in study population.

\begin{tabular}{|c|c|c|c|}
\hline & Normal & Osteopenia & Osteoporosis \\
\hline Dexa scan total & $20(26.66 \%)$ & $35(46.66 \%)$ & $20(26.66 \%)$ \\
\hline Dexa scan hip & $27(32 \%)$ & $37(49.33 \%)$ & $11(14.66 \%)$ \\
\hline Dexa scan spine & $33(44 \%)$ & $25(33.33 \%)$ & $17(22.66 \%)$ \\
\hline
\end{tabular}

The osteopenic changes in hip and spine are changes are associted with severe associated with mild derangement in derangement of markers. As shown in Tables biochemical markers and osteoporotic

Table (7): Pearsson correlation between dexa scan and calcium.

\begin{tabular}{|c|c|c|c|c|}
\hline & & T. Score HIP & T_score SPINE & Calcium \\
\hline \multirow{3}{*}{ T. Score HIP } & Pearson Correlation & 1 & $.680^{* *}$ & $-.275-^{*}$ \\
\hline & Sig. (2-tailed) & & (.000 & .017 \\
\hline & $\mathrm{N}$ & 75 & 75 & 75 \\
\hline \multirow{3}{*}{ T_score SPINE } & Pearson Correlation & $.680^{* *}$ & 1 & $-.273-^{*}$ \\
\hline & Sig. (2-tailed) & .000 & & .018 \\
\hline & $\mathrm{N}$ & 75 & 75 & 75 \\
\hline \multirow{3}{*}{ calcium } & Pearson Correlation & $-.275^{*}$ & $-.273^{*}$ & 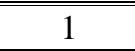 \\
\hline & Sig. (2-tailed) & .017 & .018 & \\
\hline & $\bar{N}$ & 75 & 75 & 75 \\
\hline
\end{tabular}

There is no significant correlation between the study population as shown in Table (8). parathrmon level and serum ca level among 
Mineral Derangement and Bone Diseases in Uremic Patients on hemodialysis in Ibn-Sina Hemodialysis

Table (8): Sperman s rho correlation between PTH level and serum ca level.

\begin{tabular}{|c|c|c|c|c|}
\hline & & & PTN & $\mathbf{C a}$ \\
\hline \multirow{6}{*}{ Spearman's rho } & \multirow{3}{*}{ _PTN } & Correlation Coefficient & 1.000 & .190 \\
\hline & & Sig. (2-tailed) & . & .103 \\
\hline & & $\mathrm{N}$ & 75 & 75 \\
\hline & \multirow{3}{*}{ Calcium } & Correlation Coefficient & .190 & 1.000 \\
\hline & & Sig. (2-tailed) & .103 & . \\
\hline & & $\mathrm{N}$ & 75 & 75 \\
\hline
\end{tabular}

There is a significant correlation between correlation between po4 and ca,or vit $\mathrm{D}$ as PTH level and PO4 level and no significant shown in Table (9).

Table (9): Sperman s rho correlation between phosphate level and parathyroid hormone, vit D, and calcium.

\begin{tabular}{|c|c|c|c|c|c|}
\hline Spearman's rho & & PTN & VIT D & CA & PO4 \\
\hline $\mathrm{PO} 4$ & Correlation coefficient & $.340^{* *}$ & .001 & -.107 & 1.000 \\
\hline & Sig. (2-tailed) & .003 & .994 & .361 & . \\
\hline & $\mathrm{N}$ & 75 & 75 & 75 & 75 \\
\hline
\end{tabular}

There is a very significant correlation hip and spine area as shown in Table (10). between vit $\mathrm{D}$ level and dexa results in both

Table (10): Correlations Vitamin D T score spine and hip.

\begin{tabular}{|c|c|c|c|c|}
\hline & & T.Scor HIP & T_score SPINE & Vit D \\
\hline \multirow{3}{*}{$\begin{array}{c}\text { T. Score } \\
\text { HIP }\end{array}$} & Pearson Correlation & 1 & $.680^{* * *}$ & $-.577-^{* * 1}$ \\
\hline & Sig. (2-tailed) & & .000 & .000 \\
\hline & $\mathrm{N}$ & 75 & 75 & 75 \\
\hline \multirow{3}{*}{$\begin{array}{l}\text { T_score } \\
\text { SPINE }\end{array}$} & Pearson Correlation & $.680^{* * *}$ & 1 & $-.329-^{* *}$ \\
\hline & Sig. (2-tailed) & .000 & & .004 \\
\hline & $\mathrm{N}$ & 75 & 75 & 75 \\
\hline \multirow{3}{*}{ vitD } & Pearson Correlation & $-.577^{* *}$ & $-.329-^{* *}$ & 1 \\
\hline & Sig. (2-tailed) & .000 & .004 & \\
\hline & $\mathrm{N}$ & 75 & 75 & 75 \\
\hline
\end{tabular}

*Correlation is significant at the 0.01 level (2-tailed).

There is a great predictable value that can be obtained from mineral analysis for patient with mineral and bone diseases and used as a good indicator for follow up of them prior to do dexa scan and can be depend on these biochemical markers as screening tests to evaluate the bone mineral diseases especially when dexa scan is not available in some peripheral hospitals or areas as shown in Table (11). 
Mineral Derangement and Bone Diseases in Uremic Patients on hemodialysis in Ibn-Sina Hemodialysis Center / Diyala

Table (11): Spearman s rho correlation between mineral score (biomarkers) and dexa scan Correlations between mineral score and dexa scan.

\begin{tabular}{|c|c|c|c|c|}
\hline & & & Mineral Score & Dexa scan \\
\hline \multirow{6}{*}{ Spearman's rho } & \multirow{3}{*}{ Mineral Score } & Correlation Coefficient & 1.000 & $.861^{* * *}$ \\
\hline & & Sig. (2-tailed) & & 2.000 \\
\hline & & $\mathrm{N}$ & 75 & 75 \\
\hline & \multirow{3}{*}{ Dexa scan } & Correlation Coefficient & $.861^{* * *}$ & 1.000 \\
\hline & & Sig. (2-tailed) & .000 & \\
\hline & & $\begin{array}{ll}\mathrm{N} \\
\end{array}$ & $\overline{775}$ & $\overline{775}$ \\
\hline
\end{tabular}

There is a very significant Anova regression between mineral (biomarkers) and dexa scan results for future relation and it is dependable Table (12).

Table (12): Anova a regression between the mineral score and dexa scan results.

\begin{tabular}{|c|c|c|c|c|c|}
\hline Model & Sum of Squares & df & Mean Square & $\mathrm{F}$ & Sig. \\
\hline Regression & 13.395 & 1 & 13.395 & 183.615 & $.000^{\mathrm{b}}$ \\
\hline Residual & 5.325 & 73 & .073 & & \\
\hline Total & 18.720 & 74 & & & \\
\hline
\end{tabular}

\section{Discussion}

The chronic kidney disease is a worldwide public health problem associated with high morbidity and mortality [14]. Secondary hyperparathyroidism after HD has been reported even in the presence of excellent HD [15]. In this study, CKD on HD programme, calcium, phosphorus and PTH considerably normalized in majority of the patients.

\section{Age distribution}

The mean ages of our patients 54.5yrs ,the common age groups affected 50-59 y are $27(36 \%)$,then age group 60-69 y were $17(22.7 \%)$.there is significant correlation between age and hyper tension $(\mathrm{p}=.040)$ and with $\operatorname{BMI}(\mathrm{p}=.000)$.

Our study is agree by a study done by others 54.5 and 50.0 years respectively [16]. In our study, the Anova table between age groups and biochemical markers is highly significant $(p-$ value $=.002)$ while not within the same age groups but only for the PTH .There was a statistically significant relationship between increased age of the patients as well as duration of dialysis and a high PTH level $(\mathrm{p}<0.001)$.

A study done by Sidney Klawansky, et al., showed that there are links between osteoporosis increased mortality, possibly reflecting links of low BMD with poor nutritional status and vascular calcification[16].

\subsection{Gender}

In our study, males made up the majority of the patients with51 (68\%) and females $24(32 \%)$ this goes with other study done in Africa (Nigeria, Senegal and Burkina Faso) have also reported a male preponderance 
among patients with ESRD (19).this is due to many causes one of them the common causes of uremia are DM and hypertension which more in male than in female The mean(SD) of PTH in males were 149 .5(+_252.2) while in females is 137,3.(-+266.1)The vitamin D in males with mean of 39.59(_+13.27) and in females 34.73(+_8.632).Ca in males with mean 2.002(_+.888) were as in females was $2.054(-+.377)$.the po4 was $2.25(-+1.45)$ and $2.1(-+1.44)$ in male and females respectively while alkaline phosphatase nearly equal with $123.4(-+90.48)$ and 122.9(-+79.27)for them respectively. The T-score mean in males is 1.27 SD and for females -1, 62 SD similar to what was reported in other studies[13,17,18, ].Which agree with many studies. In one study by $M$ Asaka, H Iida, M FUJITA, K IZUMINO, M TAKATA, H SETO, and S SASAYAMA, female was inversely correlated with BMD with age, but not in male HD patients [20]. Which may be due to hormonal changes and exercise reduction with chronic diseases. Female gender has also been linked as an independent risk factor.

\subsection{BMI}

The majority of patients in our study were overweight This possibly because many patients have fluid overload and didn't reach the dry weight The higher level of PTH mean was with overweight group $176.5(-+308.5)$ and lowest with normal weight 94.44(+127.6 ) also major reduction of vit $\mathrm{D}$ occur in over weight group 35.11(-+8.46) beside highest po4 mean $2.36(-+1.52)$ and lowest in normal weight group for both 41.84(-
+13.93), 2.01(-+1.188) respectively. The alkaline phosphatase follow the same manner for over weight is highest but lowest mean values in obese 136.2(-+103.2) and 80.18(+34.91) .The T-score reduction is also associated with overweight group with mean of $-1.556(-+.946)$.

There is no significant Anova correlation between BMI with PTH,Ca,po4 and age but high significant with alkaline phosphatase $(\mathrm{p}$ value $=.005)$ and $\mathrm{T}$-score values $(\mathrm{p}$ value $=.0048$ ) in Duncan test for means correlations and also significant person $\mathrm{s}$ correlation between BMI and $\operatorname{sex}(\mathrm{p}$ value = $.0020)$ and very significant with $\mathrm{DM}(\mathrm{p}=.000$ ) which are similar to other studies results. There was a statistically significant relationship between increased age of the patients as well as duration of dialysis and a high PTH level $(\mathrm{p}<0.001)$ [18].

The study by Bess et al.,showed there is a positive correlation between body weight and BMD in the general population [22]. Similarly, several studies demonstrated that the body size relates with BMD also in CKD and ESRD patients [24; 25; 26].A study done in Turkey among renal HD, low BMI values and decreased $25 \mathrm{OH}$ vitamin $\mathrm{D}$ levels were found to be a main risk factors for loss of bone mineral density [27]. Priyanka et al reported a positive correlation between BMI and bone mineral density in spine and femur. Longer CKD duration prior to HD showed greater loss of BMD in the femur[28].

In general body mass index (BMI) associates with BMD or bone mass as measured by DEXA and the BMI is a 
predictor of BMD also in ESRD patients [29]. Thus, not surprisingly, body weight and BMI were primary responsible factors for BMD variation, osteoporosis and fractures (30.31).

\section{Socio economic status}

In present study, the patients were distributed as medium status in $48((61.33 \%)$ low status 22(29.33) and high status in $5(6.6 \%)$ and this show only significant Duncan test correlation with p04 mean $(\mathrm{p}=.002)$ but no significance with PTH, ca, vit $\mathrm{D}$, or $\mathrm{T}$-score. Similar results were seen in another study [32].

This agree the study done by Catherine $\mathrm{O}$ Stehman-Breen, Donald J Sherrard, Astier M Alem Daniel L Gillen Susan R Heckbert, Craig S Wong, Adrianne Ball, and Noel S Weiss [30].

\section{Hypertension and diabetes mellitus}

Hypertension in my study was present in 61 (81\%) patients distributed as males 44 (58.7\%) and females 17 (22.7\%) followed by diabetes mellitus in $25(33.33 \%)$ patients distributed as males $18(24 \%)$ and females $7(9.3 \%)$ patients. There is no significant correlation between hypertension and other (ca, PTH, po4, vit D, T-score. Serum albumin nor with Alp). There is no significant Pearson s correlation between hyper tension and age ( $\mathrm{p}$ value $=.235)$ ) while the significant person s correlation is between the DM and age ( $\mathrm{p}$ value $=.040)$. And with vit $\mathrm{d}(\mathrm{p}=.007)$.

This is in contrast to the studies done in Japan and Iran done by Pieter E, Kathleen C, Dirk $\mathrm{K}$ et al. Where diabetes was reported as etiology of kidney disease in $26.5 \%$ and
$78.6 \%$ of the study subjects respectively while in Iran hypertension was reported in 23.6\% [33,34]. In Europe and United States, diabetic nephropathy constitutes about 50\% of the causes of ESRD while in Africa the predominant causes are hypertension and CGN thesis higher than our study.

Presentation of ESRD in Africa is often late and diagnosis prior to presentation is often inaccurate without the recognition of renal failure by the referring unit. In a study of Nigerian patients, the etiology of renal failure was undetermined in $62 \%$ and of the remaining patients whose etiology was ascertained, hypertension accounted for $61 \%$ which is agree with our study, while in diabetes mellitus for $11 \%$ and CGN for $5.9 \%$ (102)is lower than our study.

Other associated diseases (genitourinary Renal. Stones, bone diseases. Fractures), Steroid and NSAID drugs: There is significant ANOVA correlation between urinary diseases and phosphate $(\mathrm{p}=.067)$ and between renal stones and serum albumin $(\mathrm{p}=.078)$.also between NSAID and s.albumen ( $\mathrm{p}=.091)$.

\section{Hyperparathyroidism}

In present study which comprised of 75 ESRD on HD ,hyperparathyroidism was found in 31 (41.33\%) patients, and the majority were having normal acceptable value of PTH 43(57.33\%) with mean(-+SD) of 145.6(-+255.16) at a total values while distributed as highest level with 192.5(+320.6) for duration of dialysis of $4-6$ months and 31.64(-+38.38)the lowest for $7-$ 12 months duration of dialysis with only 1 
$(1.33 \%)$ has

hypoparathyroidism

.There is no significant correlation by anova tables between the groups of duration of dialysis in parathyroid levels but there is significant between the same group of duration of dialysis $(\mathrm{p}=.018)$.

The mean of PTH for males were 149.5(+252.6) and for the females 137.3(-+266.1) and the highest in age group 30-39 yrs. were $416 \mathrm{pg} . / \mathrm{ml}$ and lowest in age group of 70$79 y s \quad 10.05 \mathrm{pg} . / \mathrm{ml}$ with significant correlation by Anova table between age group ( $p$ value $=.002$ ). There is no significant between the means of age groups o by the Duncan test.

The prevalence of HPT in a cross-sectional study done by Madhumati et al [35] among 106 renal hemodialysis who were at least 6 months duration belonging to CKD stag 5 was $66 \%$. Which is higher than our study. Similar cutoff levels for defining hyperparathyroidism were used i.e. PTH> $65 \mathrm{pg} / \mathrm{ml}$. We found no correlation between HPT and duration, time on dialysis and eGFR which is similar to what was reported by Mathumati et al., Gholamhossein reported hyperparathyroidism $\quad(\mathrm{PTH}>60 \mathrm{pg} / \mathrm{ml}) \quad$ in $7.1 \%$ of the study population 3 months duration and was associated with a mean SD of $54>18.68$ months compared to a mean SD of $14.26>8.34$ months $(p<0.001)$ in those with post HD PTH of $\leq 60 \mathrm{pg} / \mathrm{ml}$. Though our patients with elevated PTH had been on dialysis for a longer time (median duration 5 months) to HPT compared to those with normal PTH (median duration 12 months) or low PTH (median duration 10 months), the difference was not statistically significant.

The prevalence of hyperparathyroidism in current study was like to that among CKD patients who were not yet on dialysis attending clinic at $\mathrm{KNH}$ done by Armando Torres, Aurelio PR, Maria TC et al, where $21.6 \%$ of the study population had HPT. These results suggest a persistence of hyperparathyroidism after starting regular HD program. Pre HD hyperparathyroidism has been associated with persistent hyperparathyroidism after HD [13].

A shorter time of non-dialysis prior to $\mathrm{HD}$ (between diagnosis and HD) may reduce the risk of persistence hyperparathyroidism after. Secondary hyperparathyroidism has been associated with metabolic bone disease which manifests as bone pain and skeletal fractures [36]. In the EVOLVE study, the use of calcimimetic agent cinacalcet in patients with moderate- to - severe secondary HPT who were undergoing hemodialysis, did not significantly reduce the risk of death or major cardiovascular events [36].

However, 1 year after HD resolution of hyperparathyroidism is not complete in $50 \%$ of HD patients. Duration of dialysis, parathyroid gland size and development of nodular/ or monoclonal hyperplasia of parathyroid gland are the most important possible factors responsible for HPT[37].

\section{Calcium level}

Almost half $39(52 \%)$ of our study population had hypocalcemia with mean calcium levels of $2.02 \mathrm{mmol} / \mathrm{l}$. Male were $29(38.7 \%)$ and females $10(13.3 \%)$, there are 
35 patients $(46.6 \%)$ have normal calcium level distributed as 22(29.3\%) males and $13(17.3 \%)$ females with mean ca level of 2.22 and only one female has hyper calcemia $(1.33 \%$. we found no significant correlation between age group nor duration of dialysis with hypocalcemia.

We found significant correlation between hypocalcemia and estimated T_score and no with BMI. Only 1(1.33\%) of our patients had hypercalcemia as it has been reported in other studies with hypercalcemia ranging from $3 \%-21 \%$ [33].

In the early post HD period serum calcium has been reported to follow a biphasic pattern with an immediate post HD decline followed by a significant increase. A low pre hemodialysis PTH has been found to be a predictor of severe Hypocalcemia post HD although no significant correlation between the level of ca and PTH [33]. Other study (34) reported a higher prevalence of low turnover bone disease $(50.9 \%)$ of the patients with biochemical evidence of mineral bone disease, than a higher turn-over disease among CKD pre-dialysis patients attending clinic at $\mathrm{KNH}$. This might help explaining presence of hypocalcemia in our population (we find 2 of normally controlled person from $25(8 \%)$ having hypocalcemia only without any other mineral or dexa abnormality) which mean that our dexa scan is a reliable test in assessment study. The effect of corticosteroids on serum calcium levels as a previous medication history among study population can never be ignored.
In this studies where hypercalcemia was reported among HD 1(1.33\%) factors that were HPT and longer duration of dialysis [ 20]. Calcium was the major regulator of the parathyroid at the levels of secretion, gene expression and cell proliferation. Studies have shown hypocalcemia to cause a profound increase in parathyroid cell proliferation, PTH gene expression and subsequent PTH synthesis [38]. Hypocalcemia among CKD patients undergoing hemodialysis has been related with increased mortality especially in the presence of hyperphosphatemia and elevated PTH levels [37]. All 100\% patients were on calcium supplements regularly despite having different calcium level because our patient compliance is less than other population and a bad minded about hemodialysis programme and poor patients and nutritional and gastrointestinal complication of uremia. In this study vitamin D levels were low determined and thus a correlation with low serum calcium levels in this population could be ascertained [11].

\section{Phoshate level}

In my study, nearly as half as $41(54.6 \%)$ of renal HD patients had high serum phosphate had high serum phosphate distributed as male $27(36 \%)$ and females 14(18.66\%) with highest level in age group30-39yrs2.5 (+1.488 ) with mean of and lowest level at age group of70-79 yrs. with means of 1.46 ($+.99011)$ Only $4(5.33 \%)$ patients equally distributed 2 male and 2 female gender $(2.7 \%)$ were found to have hypophosphatemia and another 30 (40\%) 
patients with males of 22(29.33\%)and females $8(8.33 \%)$ having normal phosphate level .No significant correlation was found between serum phosphate and different age groups or duration of dialysis ., sex, , but significant with $\mathrm{PTH}(\mathrm{p}=.035)$.Also ca and dexa scan results were not associated with significant correlations with either higher or lower levels of serum phosphate.

Serum phosphorus levels are usually elevated prior to renal dialysis and decreases rapidly following HD to within or below range for patients with normal kidney function in the first few post HD months [40].

\section{Vitamin D}

The results in my study were $73(97.33 \%)$ have vit D deficiency and 2(2.33\%) of patient were normal. There is very significant anova correlation between vit $\mathrm{D}$ and $\mathrm{t}$-score $(\mathrm{p}=$ .000 ) and also and by persons correlation also $(\mathrm{p}=.000)$ and for both hip and spine areas. But not with phosphate $(\mathrm{p}=.088)$ nor with calcium which compatible with other studies in world. This study results are go with that done by Grahame J Elder (51) but not agree by study in japan by K Nakatsuka and Y Nishizawa. Vitamin D supplementation reduces serum PTH levels and improves bone strength.

\section{Dexa (T-sore) results}

The results of our study, the dexa scan as a whole show $20(26.66 \%)$ of patients with normal BMD, and 35(46.6\%) with osteopenia BMD, and 20(26.66\%) of them having osteoporotic BMD and no fractures in between but there is considerable difference in BMD between the hip area t-score and spine area t-score.

\section{a.The hip t-score}

Shows that 27(32\%) were normal BMD with mean (SD) $-0.218 \quad(-+0.9856)$ $37(49.33 \%)$ had osteopenia with mean $1.736(+-.2938)$ and $11(14.66 \%)$ of them with osteoporosis with mean $-2.958(-+0.370)$. The osteopenia of hip associated with lower values of $\mathrm{PTH}, \mathrm{PO} 4, \mathrm{Ca}$ while osteoporotic hip associated with highest elevation of PTH, ALP and po4 but with lowest most values of $\mathrm{Ca}$, VIT D, total serum protein and albumin [39]. There is significant correlation in anova table between hip t-score and PTH $(\mathrm{p}=.000)$ and with serum albumin $(\mathrm{p}=.000)$ while no significant correlation with PTH in Duncan test nor with albumin level. In spearman $\mathrm{s}$ rho there is very significant correlation between hip T-score and serum albumin $(\mathrm{p}=.000)$ and with alkaline phosphatase $(\mathrm{p}=.004)$ and also with total serum protein $(\mathrm{p}=.000)$ and no significant correlation with $\mathrm{Ca}$ or po4.

\section{b.In spine T-score}

Appeared 33(44\%) with normal BMD with mean (SD) -0.581 (+-1.145), 25 (33.33\%) osteopenia with mean of-1.760(-+0.4500) and 17 (22.66\%) osteoporosis BMD with mean of $-1.385(-+0.857)$. The osteopic spine lower derangement in PTH, PO4, VIT D, and ALP elevations, were as osteoporotic spine associated with highest derangement in PTH, PO4 and ALP elevations and lowest value hypocalcemia. There is Significant statistical correlation between spine T-score and serum calcium, total serum protein, albumin and 
alkaline phosphatase with ( $\mathrm{p}=.003),(\mathrm{p}=.005)$, $(\mathrm{p}=.000),(\mathrm{p}=.006)$ by anova test respectively. It is not clear whether peripheral or central bone, or cortical or trabecular bone, are to be preferred for the evaluation of BMD in CKD patients.

\section{Conclusions}

1-This study demonstrated that elevated serum PTH levels are present even after HD and are associated with many factors for bone diseases. It also showed presence of low PTH levels though the prevalence is lower than those reported in the CKD counterpart. This may indicate continuing presence of a dynamic bone disease if PTH levels are used as surrogate markers for bone turnover.

2-Hypocalcemia was present in almost half of HD as opposed to hypercalcemia reported in other studies. This may be as a result of higher prevalence of a dynamic bone disease in our CKD patients. Hypocalcemia was associated with relatively older patients and was not correlated with duration of dialysis.

3-Nearly half of our patients had serum phosphorus levels within reference ranges.

4-There is great predictable value can be obtained from mineral analysis for patient with mineral and bone diseases and used as a good indicator for follow up of them without need to do dexa scan and can be depended on these biochemical markers as screening tests to evaluate the bone mineral diseases especially when dexa scan is not available in some peripheral hospitals or areas.

\section{References}

[1]Pendse S, Singh AK. Complications of chronic kidney disease: Anemia, mineral metabolism, and cardiovascular disease. Med Clin North Am. 2005;89:549-61. [2]Martin KJ, Olgaard K, Coburn JW, Coen GM, Fukagawa $\mathrm{M}$, Langman $\mathrm{C}$, et al. Diagnosis, assessment, and treatment of bone turnover abnormalities in renal osteodystrophy. Am J Kidney Dis. 2004;43:558-65.

[3]Kronenberg F, Mündle M, Längle M, Neyer U. Prevalence and progression of peripheral arterial calcifications in patients with ESRD. Am J Kidney Dis. 2003;41:1408.

[4]Martola L, Barany P, Stenvinkel P. Why do dialysis patients develop a heart of stone and bone of china? Blood Purif. 2005;23:203-10.

[5]Raggi P, Boulay A, Chasan-Taber S, Amin N, Dillon M, Burke SK, et al. Cardiac calcification in adult hemodialysis patients. A link between end-stage renal disease and cardiovascular disease? J Am Coll Cardiol. 2002;39:695-701.

[6]Norris KC, Crooks PW, Nebeker HG, Hercz G, Milliner DS, Gerszi K, et al. Clinical and laboratory features of aluminum-related bone disease: Differences between sporadic and "epidemic" forms of the syndrome. Am J Kidney Dis. 1985;6:342-7.

[7]Dawn Shao TL, Terence Yi SK, Stephenie FC et al. Prevalence and Patterns of Bone Loss in the First Year After Renal Transplant in South East Asia Patients. Transplantation 2011;92:557-563. 
[8]Jose RW, Raul GC, Eudocia R et al. Bone disease after transplantation. Clin J Am Soc Nephrol 2006; 1: 1300-1313.

[9]Daniel, W. W. Biostatistics: A Foundation for Analysis in Health Sciences. 7th edition. New York: John Wiley \& Sons. 1999.

[10]Stavroulopoulos A, Cassidy M J D, Porter CJ et al. Vitamin D Status in Renal Transplant Recipients. Am J Transplant 2007; 7: 2546-2552.

[11]Taziki O, Espahbodi F, Alizadeh Forutan M et al. 25-Hydroxyvitamin D Deficiency in Kidney Transplant Recipient. Iran J Kidney 2011; 5 (1): 57-62.

[12]Levey AS, Bosch JP, Lewis JB et al. A more accurate method to estimate glomerular filtration rate from serum creatinine: a new prediction equation. Modification of Diet in Renal Disease Study Group. Ann Intern Med 1999; 130 (6): 461-70.

[13]Dousdampanis P, Trigka K, Fourtounas $\mathrm{C}$ et al. Evolution of secondary hyperparathyroidism one year after successful renal transplantation. Hippokratia 2011.15(2): 30-32.

[14] Grotz WH,Mundinger FA, Goged B et al.Bone mineral density after kidney transplantation 1995;59:982-986.

[15]Rojas E, Carlini RG, Clesca P et al. the pathogenesis of osteodystrophy after Renal Transplantation as Detected by Early Alterations in bone Remodelling.Kidney Int 2003;63:1015-1023.

[16]Sidney Klawansky, Eugene Kom Paul F Cavanaugh Jr, David Y.relationship age ,renal function and bone mineral density in the US population Osteoporosis international,14(7):570\{576,2003\}.

[17]Kawarazaki H,Shibagaki Y, Fukumoto S et al,Natural history of mineral and bone disorders after living-donor kidney transplantation. A one year prospective observational study. Therapeutic apharesis and dialysis 2011;15(5):481-487.

[18]Gholamhossein RO, Mohamad $\mathrm{HD}$, mohammadS et al.predictive factors for persistent hyperparathyroidism after kidney transplantation.Arch Iranian Med 2005;8(4):295-299.

[19]Naicker S. End-stage renal disease in Sub- Saharan and South Africa. Kidney Int Suppl. 2003;83: S119-22.

[20]M Asaka, $\mathrm{H}$ Iida, $\mathrm{M}$ FUJITA, K IZUMINO, M TAKATA, H SETO, and S SASAYAMA. Total and regional bone mineral density by dual photon absorptiometry in patients on maintenance hemodialysis. Clinical nephrology, 38(3):149\{153, 1992.

[21]Ugur A, Guvener N, Isklar I et al. Osteoporosis after Renal Transplantation: Single Center Experience. Transplantation 2001;71:645-649.

[22]Bess Dawson-Hughes, Clanton Shipp, Laura Sadowski, and Gerard Dallal. Bone density of the radius, spine, and hip in relation to percent of ideal body weight in postmenopausal women. Calcied tissue international, 40(6):310\{314, 1987.

[23] Marcel J, Cambe B, Francisco J et al. Bone Mineral Density of 704 Amateur Sportsman Involved in Different Physical Activities. Osteoporos Int 2001;12:152-157. 
[24] Abderrahmane Ghazali, Franck Grados, Roxana Oprisiu, Delia Bunea, Philippe Morini_x0012_ere, Najeh El Esper, Isabelle El Esper, Michel Brazier, Jean Claude Souberbielle,Albert Fournier, et al. Bone mineral density directly correlates with elevated serum leptin in haemodialysis patients. Nephrology Dialysis Transplantation, 18(9):1882\{1890, 2003.

[25]Maarten W Taal, Tahir Masud, Desmond Green, and Michael JD Cassidy. Risk factors for reduced bone density in haemodialysis patients. Nephrology Dialysis Transplantation, 14(8):1922\{1928, 1999. [26]Fettah Fevzi Ersoy, Stauros Ploumis Passadakis, Paul Tam, Evaggelos Dimitros Memmos, Pericles Konstantinos Katopodis, $\mathrm{C}$ etin $\square$ Ozener, Fehmi Akcicek, Taner C amsar_x0010_, Kenan Ates, Rezzan Ataman, et al. Bone mineral density and its correlation with clinical and laboratory factors in chronic peritoneal dialysis patients. Journal of bone and mineral metabolism, 24(1):79\{86,- 2006\}.

[27] Unal A, Kocyigit L, Sipahioglu MH et al. Loss of Bone Mineral Density in Renal Transplantation Recipients. Transplant Proc. 2010; 42(9):3550-3.

[28] Priyanka G, George A, Balaji P et al. Bone Mineral Disease in Renal Transplantation- An Indian Experience. JNRT 2009; 2(1): 63-70.

[29]Marianne Relle Andreassen, Peter Eskildsen, Bente Langdahl, and KlausOlgaard. Bone mineral density and biochemical markers of bone turnover in patients with predialysis chronic renal failure. Kidney international, 56(3):1084\{ 1093, 1999.

[30]Catherine O Stehman-Breen, Donald J Sherrard, Astier M Alem, Daniel L Gillen, Susan R Heckbert, Craig S Wong, Adrianne Ball, and Noel S Weiss. Risk factorsfor hip fracture among patients with end-stage renal disease. Kidney interna- tional, 58(5):2200\{2205, 2000.

[31] Shu-Feng Lei, Fei-Yan Deng, Miao-Xin Li, Volodymyr Dvornyk, and Hong-Wen Deng. Bone mineral density in elderly chinese: e ects of age, sex, weight, height and body mass index. Journal of bone and mineral metabolism, 22(1):71\{78,2004\}.

[32]Csaba A. Bone mineral disorders in patients on maintainance hemodialysis and after kidney transplantation: MD Thesis, 2009. Semmelweis University. Budapest.

[33]Pieter E, Kathleen C, Dirk K et al. Natural History of Parathyroid Function and Calcium Metabolism after Kidney Transplantation. Nephrol Dial Transplant 2004;19:1281-1287.

[34]Kawarazaki H, Shibagaki Y, Fukumoto $S$ et al, Natural History of mineral and bone disorders after living-donor kidney transplantation. A one year prospective observational study. Therapeutic Aphesis and Dialysis 2011; 15(5): 481-487.

[35]El Maghraoui, Borderie et al. 1999; Maillefert, Aho et al. 2001; El Maghraoui 2004; El Maghraoui 2004; El Maghraoui, Do Santos Zounon et al. 2005.

[36] Armando Torres, Aurelio PR, Maria TC et al, Parathyroid function in long-term renal 68 transplant patients: importance of pre- 
transplant PTH concentrations. Nephrol Dial

Transplant 1998;13(3):84-97.

[37]Miller JE, Kovesdy CP, Noris KC, et al. Association of cumulatively low or high serum calcium levels with mortality in long term hemodialysis patients. Am J Nephrol 2010;32: 4003-413.

[38]Stavroulopoulos A, Cassidy M J D, Porter CJ et al. Vitamin D Status in Renal Transplant Recipients. Am J Transplant 2007; 7: 2546-2552.

[39]El-Amm JM, Doshi MD, Singh A, et al. Preliminary experience with cinacalcet use in persistent secondary hyperparathyroidism after kidney transplantation. Transplantation 2007; 83(5):546-9.

[40]Kamar N, Gennero I, Spataru L, et al. Pharmacodynamic effects of cinacalcet after kidney transplantation: once-versus twicedaily dose. Nephrology Dialysis Transplantation 2008; 23:3720-4. 